\title{
Numerical Method for Exposure Assessment of Wireless Power Transmission under Low-Frequency Band
}

\author{
Minhyuk Kim ${ }^{1}$, SangWook Park ${ }^{2 *}$, and Hyun-Kyo Jung ${ }^{1}$ \\ ${ }^{1}$ Department of Electrical and Computer Engineering, Seoul National University, Seoul 08826, Korea \\ ${ }^{2}$ ICT Convergence Research Team, EMI/EMC R\&D Center, Corporation Support \& Reliability Division, \\ Korea Automotive Technology, Choenan 31214, Korea
}

(Received 9 March 2016, Received in final form 11 August 2016, Accepted 31 August 2016)

\begin{abstract}
In this paper, an effective numerical analysis method is proposed for calculating dosimetry of the wireless power transfer system operating low-frequency ranges. The finite-difference time-domain (FDTD) method is widely used to analyze bio-electromagnetic field problems, which require high resolution, such as a heterogeneous whole-body voxel human model. However, applying the standard method in the low-frequency band incurs an inordinate number of time steps. We overcome this problem by proposing a modified finitedifference time-domain method which utilizes a quasi-static approximation with the surface equivalence theorem. The analysis results of the simple model by using proposed method are in good agreement with those from a commercial electromagnetic simulator. A simulation of the induced electric fields in a human head voxel model exposed to a wireless power transmission system provides a realistic example of an application of the proposed method. The simulation results of the realistic human model with the proposed method are verified by comparing it with the conventional FDTD method.
\end{abstract}

Keywords : finite-difference time-domain method, hybrid technique, dosimetry, wireless power transmission, surface equivalence theorem

\section{Introduction}

Wireless power transmission (WPT) technique has been studied narrowly due to technical difficulties, however, research for commercialization is in the process actively in accordance with the development of non-radiative WPT technique with Marin Soljačić research group at Massachusetts Institute of Technology [1]. Therefore, prior to the advancement and commercialization of this technology, there is an essential need for research on the adverse health effect when the human body is exposed to electromagnetic field (EMF).

The EMF assessment throughout the experiment can be applied only to the homogeneous medium and have a limited frequency range from $150 \mathrm{MHz}$ to $9 \mathrm{GHz}$. Recently, numerical EMF assessment is conducted using anatomical whole-body voxel model regardless of frequency ranges.

(C)The Korean Magnetics Society. All rights reserved.

*Corresponding author: Tel: +82-41-559-3114

Fax: +82-41-559-3068, e-mail: parksw@katech.re.kr
The FDTD method is one of the best methods in terms of computer time and memory, as it does not require matrix calculation process, because it is an explicit method. Therefore, this method is very well suited to problems that require high resolutions such as human model. Furthermore, this method is applicable to problems involving heterogeneous dielectric and lossy materials, unlike Method of Moments (MoM) whose technique is not suitable to interpret those materials. Hence, it is the most widely used method for bio-electromagnetic applications, which exceed a few MHz [2].

However, the standard FDTD method has a problem when calculating at low frequencies. Numerous time steps are needed, which leads to excessively long computation times to converge to a steady state. In order to solve this problem, implicit-FDTD schemes [3-5] and several numerical methods with quasi-static approximation (QSA) have been researched. The implicit FDTD methods are theoretically unconditionally stable. However, this method still presents problems under a few $\mathrm{MHz}$ because the acceleration factor reduces the simulation time as well as the accuracy when the factor exceeds the limit. 
There have been several studies of numerical methods with QSA researched for the low-frequency band problems such as an impedance network method [6, 7], current vector potential method [8], and a scalar potential finite-difference method [9]. The incident magnetic field problem is easily simulated by the above methods as it can be calculated by Faraday's law. However, an incident electric field problem is not easy to simulate as the charge on the surface of the target must be known to analyze the electrical coupling. There is a research that induced electric field is not always meaningless amount when computing the dosimetry of the human body model under low frequencies [10]. Therefore, there is a need to devise a technique to compute both electric and magnetic fields easily.

The frequency-scaling FDTD method was first introduced [11]. The method interprets the problem at a higher frequency than the frequency of the target. Then, the obtained result is converted by the ratio of the previous frequency relative to that of interest. This method can dramatically reduce the simulation time of a low-frequency problem; however, the following problems can nonetheless arise: i) the FDTD method still requires a substantially amount of simulation time at frequencies of few $\mathrm{MHz}$, and ii) errors occur as the skin depth is not negligible at the frequency used in the original paper. The quasi-static FDTD (QS-FDTD) method [12] clear up this problem using a ramp source. However, it mainly focused on the plane wave excitation. When directly applying this method to conductor or/and dielectric models, i.e., antennas, which radiate electromagnetic fields, calculation of the radiation characteristics of the antenna cannot be done.

It is difficult to obtain a response of low frequency band electromagnetic field problems by the above-mentioned problems. Therefore, we propose a new approach, i.e., an FDTD method with quasi-static source approximation using the surface equivalent theorem. We refer to the proposed method as the surface-equivalence-theorembased quasi-static FDTD (SQ-FDTD). The surface equivalent theorem is applied to compute arbitrary source excitation, and the QSA is used to converge quickly under low frequencies in the proposed method. The method, taking into account both electric and magnetic coupling, analysis inhomogeneous complex-shaped problem in the low frequency exceptionally quickly by arbitrary source excitation. In this study, the currents on the equivalent surface by the radiation of antennas are calculated by numerical method in advance. Then, the entire field response is obtained by applying the results of the previous step to the QS-FDTD method. To verify our approach, the EMF assessment of the simplified human phantom model for the WPT system was conducted by using the proposed method. The results are compared with those of a commercial software package (FEKO [13]). Lastly, the realistic human head voxel model is analyzed by the proposed method and the results are compared with those from the standard FDTD method.

\section{The Proposed Method}

The basic concept of the proposed method is hybrid of the surface equivalent theorem and QS-FDTD method to interpret the low-frequency problem quickly by exciting arbitrary source.

The proposed method is essentially a two-step method, whereas the standard FDTD solve the problem at once, as the flowchart shown in Fig. 1. The first step of the method involves selecting arbitrary surface surrounding sources and/or scatterers and calculating currents on these surfaces. In the next step, the electromagnetic field responses are calculated using the QS-FDTD method. The results of the first step are used as a source in the second step. We split the procedure to apply the QSA to currents on the equivalent surface. The following are detailed explanations of each part.

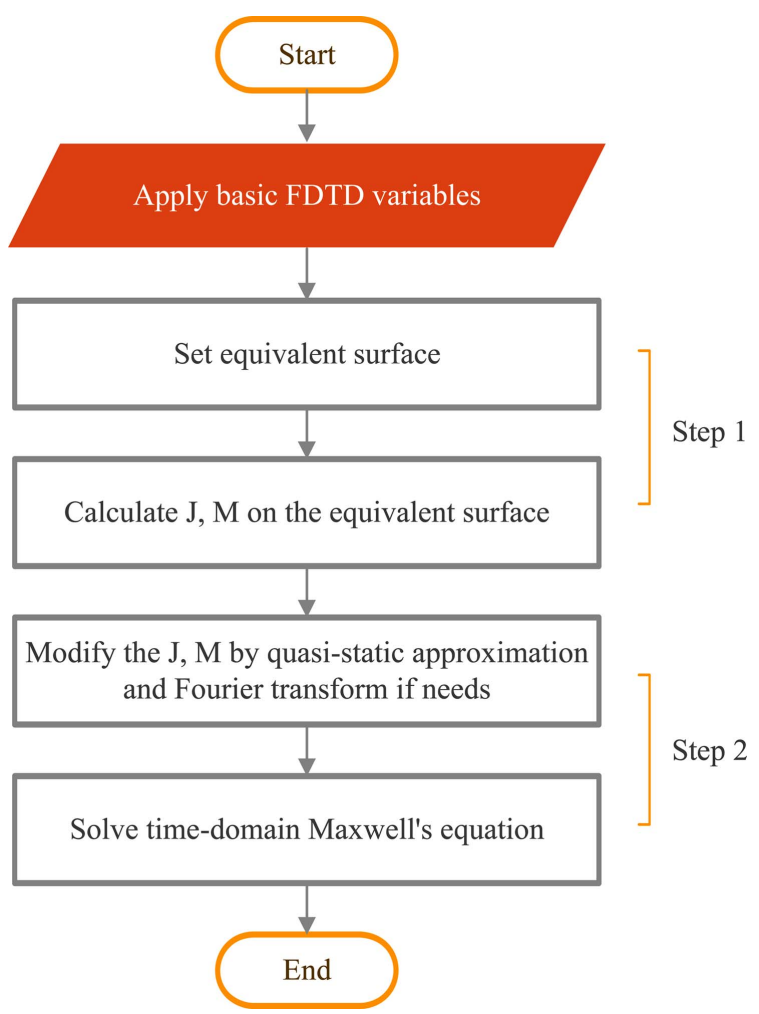

Fig. 1. (Color online) Flowchart of the proposed method. 


\subsection{Surface equivalence theorem}

In the first step of the proposed method, we extract equivalent currents on the arbitrary surface, including the sources and/or scatterers using the surface equivalence theorem.

The surface equivalence theorem was introduced in 1936 and is now widely applied in electromagnetic problems. The theorem is used to obtain the electromagnetic scattering of complex scatterers by replacing actual sources such as antennas or scatterers with fictitious surface currents on a surrounding closed surface [14].

The electromagnetic field traveling to the outside of the boundary surface $\mathrm{S}$ is computed from the surface current on the boundary surface $\mathrm{S}$. The equivalent surface is selected to enclose all complex scatterers and source. Generally, the surface has a simple shape for easy calculation, such as circular, rectangular, cylindrical, as shown in Fig. 2(a). In this study, the equivalent surface is chosen as a rectangular box, as it is easy to incorporate this into the FDTD method.

The currents on the equivalent surface is introduced to satisfy the boundary conditions, as expressed in (1) and in Fig. 2(b).

$$
\begin{aligned}
& \vec{M}_{s}=\left(\vec{E}_{2}-\vec{E}_{1}\right) \times \hat{n} \\
& \vec{J}_{s}=\hat{n} \times\left(\vec{H}_{2}-\vec{H}_{1}\right)
\end{aligned}
$$

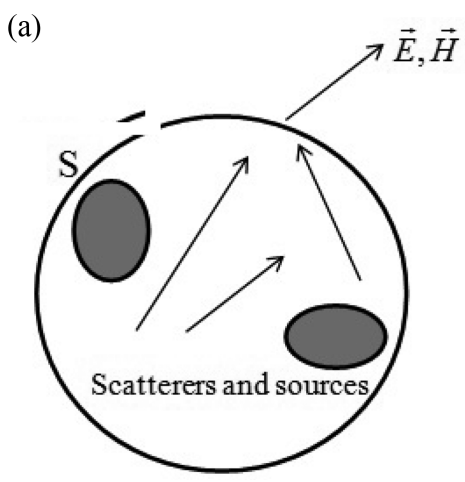

(b)

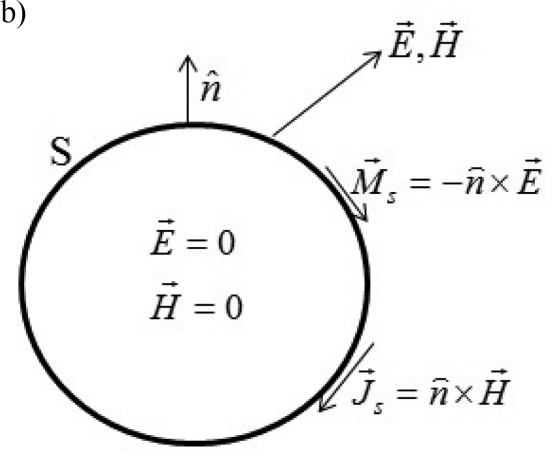

Fig. 2. Surface equivalence theorem: (a) Original problem, (b) Equivalent problem for the region outside S.
Here, $\hat{n}$ is a normal vector that penetrates the defined surface $\mathrm{S}, \vec{M}_{s}$ is the surface-magnetic current density and $\vec{J}_{s}$ is the surface-electric current density. $\vec{E}_{1}$ and $\vec{H}_{1}$ are the field outside an equivalent surface, and $\vec{E}_{2}$ and $\vec{H}_{2}$ are the field inside an equivalent surface.

The currents on the equivalent surface are determined by any theoretical or numerical technique. Any type of numerical technique can be used to the determine currents on an equivalent surface. Generally, a wire antenna is simulated by the MoM and a complex inhomogeneous configuration is calculated by a finite element method or FDTD method. Simple Fourier transform is applied to the case that the obtained currents are in the frequency domain as expressed in (2).

$$
\vec{J}_{s}=\vec{J}_{s 0} \sin (\omega t+\phi)
$$

These currents in the time domain are inserted to the QS-FDTD method as a source.

\subsection{FDTD method with QSA}

In the second step of the proposed method, we obtain the internal electric fields of human phantom excited by the electromagnetic fields from equivalent currents in the previous step by using FDTD method with QSA.

The FDTD method is a numerical analysis technique, which discretized the time-dependent Maxwell's equations using central-difference approximations to the space and time partial derivatives. To obtain a steady state sinusoidal behavior, the standard FDTD method requires a simulation time of a few periods of the frequency of interest. In the method, the time step is determined by the well-known "Courant Condition" [15]:

$$
\Delta t \leq \frac{\Delta x}{\sqrt{n} \cdot c_{0}}
$$

where $n$ is the dimension of the simulation, $c_{0}$ is the speed of light, and $\Delta x$ is the mesh size.

As the frequency goes down, the period gets longer and the number of time steps increases. Since simulation for a few periods is required, it is impossible to solve the problem with this method under a few $\mathrm{MHz}$. It is extremely time-consuming given its need for a large number of iterative simulation steps to reach steady-state. In the proposed method, the QSA is applied to the obtained currents (2) in the previous step to overcome this problem. Quasistatics is a regime where the system is small compared with the electromagnetic wavelength associated with the dominant time scale of the problem [16]. Thus, the propagation effects could be negligible in the QSA. The QSA approach is often assumed up to a few tens of megahertz [17]. Thus, quick convergence is possible 
considering the fact that the phase is known in the steadystate QSA [17, 18]. That is to say, the exterior fields of the object have the same phase as the incident field, and the interior fields of the target are first-order fields that are proportional to the time derivative of the incident field [12]. Hence, the exterior fields will have a linear behavior and the interior fields will have a constant behavior when a ramp function is applied in the incident field. Thus, the whole electromagnetic field response is quickly obtained as it is sufficient to record two successive field values after the transient response has finished [12, 19, 20].

The function used in this method was altered to start smoothly such that it avoids high-frequency contamination. For example, the time domain electric current (2) is modified as follows [12]:

$$
J_{s}=\left\{\begin{array}{cc}
0 & -\infty<t<t_{0} \\
(t-(\tau / \pi) \sin (\pi t / \tau)) / 2 & t_{0}<t<\tau \\
t-\tau / 2 & t>\tau
\end{array}\right.
$$

Here, $\tau$ is a constant that represents the functional behavior of the currents.

The magnetic current is obtained in a similar way. Finally, the approximated currents represent original field generated by the source are obtained. These currents are inserted in the time-domain Maxwell equations as expressed in (5).

$$
\begin{aligned}
& \nabla \times \vec{E}=-\frac{\partial \vec{B}}{\partial t}-\vec{M}_{s} \\
& \nabla \times \vec{H}=\frac{\partial \vec{D}}{\partial t}+\vec{J}_{s}
\end{aligned}
$$

All of the electromagnetic fields are calculated by the standard FDTD scheme. The electromagnetic field response is calculated extremely faster than they are with standard method, as the quasi-static system reaches convergence in much less than one full period.

Generally, iteration process is required in the hybrid method for considering the interaction between the methods. However, the interaction between dielectric material computed by the QS-FDTD method and the equivalent currents obtained by the numerical technique is not included in the proposed method as the effect could be omitted to speedy computation in case that the effect of the interaction is negligible [10].

\section{Method Verification}

In this paper, the currents on the equivalent surface $S$ of the rectangular box including the antennas are obtained by MoM in the first step. The next step is converting fre-

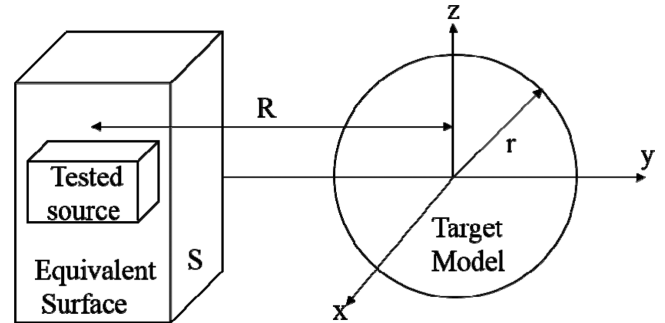

Fig. 3. System for calculating the induced electric field of a dielectric sphere from antenna radiation.

quency-domain currents into the time domain by Fourier transform (2). Then the whole electromagnetic fields are calculated by solving the standard FDTD scheme with employing the approximated currents as a source.

The simulation system, which is composed of the target model and equivalent surface, including sources and scatterers, is illustrated in Fig. 3.

The simulations were conducted to verify the proposed method by obtaining induced field exposure of a dielectric sphere with a loop, helical, and WPT system, as shown in Fig. 4.

A dielectric sphere as a simplified human phantom model has conductivity $\sigma=0.3 \mathrm{~S} \cdot \mathrm{m}^{-1}$ and a relative dielectric constant $\varepsilon_{r}$ of 30 . The operating frequency is 1 $\mathrm{MHz}$ in this paper.

The first test is conducted with a circular loop antenna whose diameter $D_{L}$ is $60 \mathrm{~mm}$.

The center of the dielectric sphere with a radius $r$ of $200 \mathrm{~mm}$ and an equivalent surface with $16 \times 16 \times 16$ are placed on the y-axis. The loop antenna is placed in the center of the equivalent rectangular box. The distance $R$ between the center of the equivalent surface and the sphere is $800 \mathrm{~mm}$. The domain is constructed by $80 \times 80 \times 80$ cubical cells $\Delta=\Delta x=\Delta y=\Delta z=20 \mathrm{~mm}$. The sinusoidal function is converted by (4) with $\tau=100 \delta t$ and $\delta t=33.3 \mathrm{ps}$.

The proposed method converged after 600 time steps. The results comparison is summarized in Table 1 between two methods, which is made based on maximum and

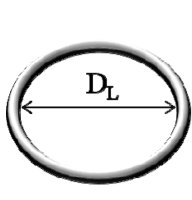

(a)

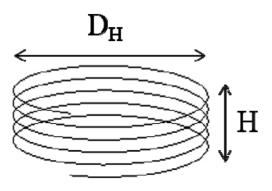

(b)

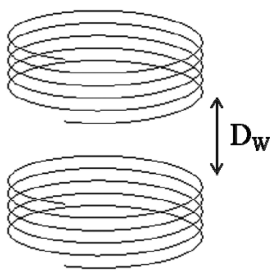

(c)
Fig. 4. Tested sources: (a) loop antenna, (b) helical antenna, and (c) WPT system. 
Table 1. Induced electric field comparisons for the loop antenna.

\begin{tabular}{lccc}
\hline \hline & \multicolumn{3}{c}{ Electric field } \\
\cline { 2 - 4 } & Plane $\mathrm{x}=0$ & Plane $\mathrm{y}=0$ & Plane $\mathrm{z}=0$ \\
\hline Maximum difference (\%) & 21.85 & 22.65 & 22.29 \\
Average difference (\%) & 8.65 & 8.87 & 8.24 \\
\hline
\end{tabular}

average relative difference as expressed in (6) and (7), respectively.

Maximum difference=Max $\left(\left|\frac{\text { FEKO-Proposed method }}{\text { Proposed method }}\right| \times 100\right)$

Average difference $=\frac{\sum_{m=1}^{n}\left|\frac{\text { FEKO-Proposed method }}{\text { Proposed method }}\right| \times 100}{n}$

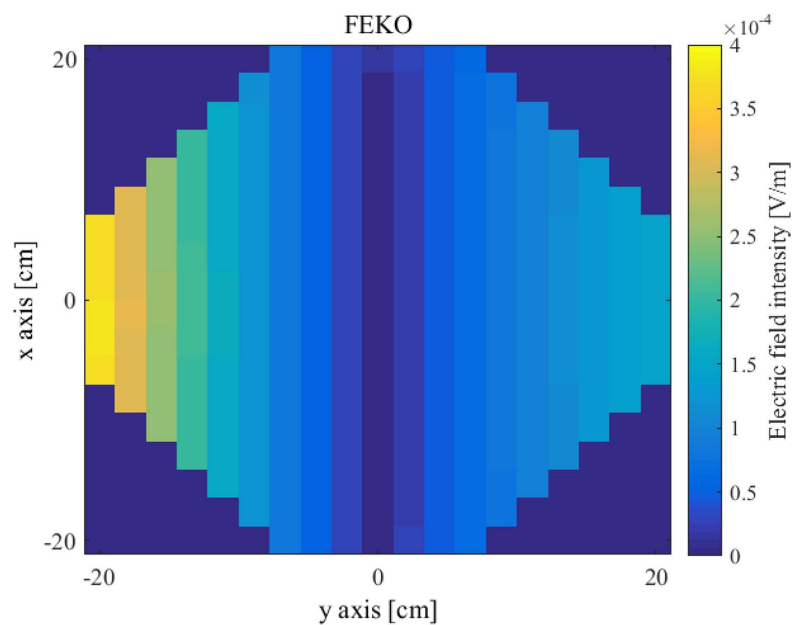

(a)

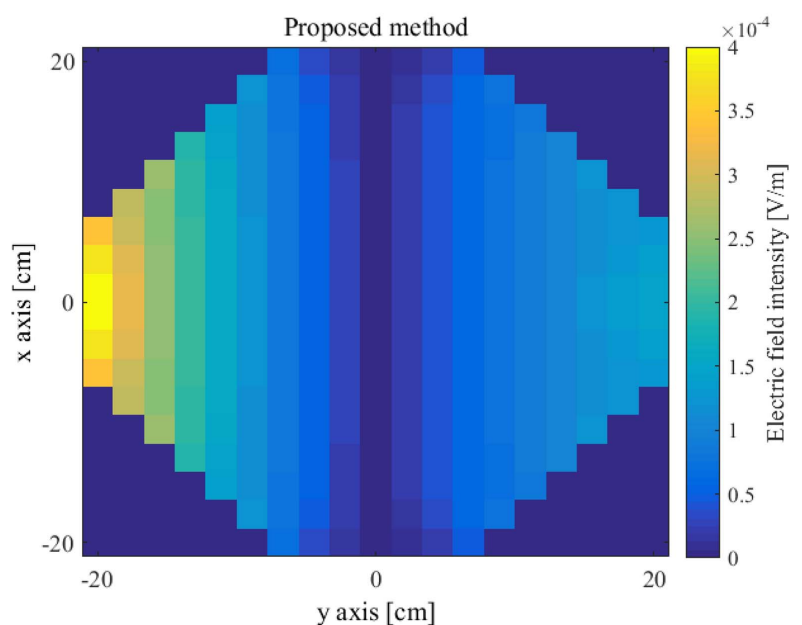

(b)

Fig. 5. (Color online) Absolute internal electric field crosssections from the loop antenna in the plane $\mathrm{z}=0$ : (a) FEKO, (b) the proposed method.
Table 2. Induced electric field comparisons for the helical antenna.

\begin{tabular}{lccc}
\hline \hline & \multicolumn{3}{c}{ Electric field } \\
\cline { 2 - 4 } & Plane $\mathrm{x}=0$ & Plane $\mathrm{y}=0$ & Plane $\mathrm{z}=0$ \\
\hline Maximum difference (\%) & 30.02 & 30.95 & 30.48 \\
Average difference (\%) & 9.87 & 11.25 & 10.33 \\
\hline
\end{tabular}

The internal electric field results obtained by the proposed method and FEKO are shown in Fig. 5.

The next simulation is with a helical antenna with a diameter $\mathrm{D}_{\mathrm{H}}$ of $60 \mathrm{~mm}$ and a height $\mathrm{H}$ of $50 \mathrm{~mm}$. The other parameters, including equivalent surface, central distance, mesh, sphere, and source properties, were identical to those used in the loop antenna test. Quick convergence is also achieved, as in the previous test, and the results of the proposed method are well matched to those

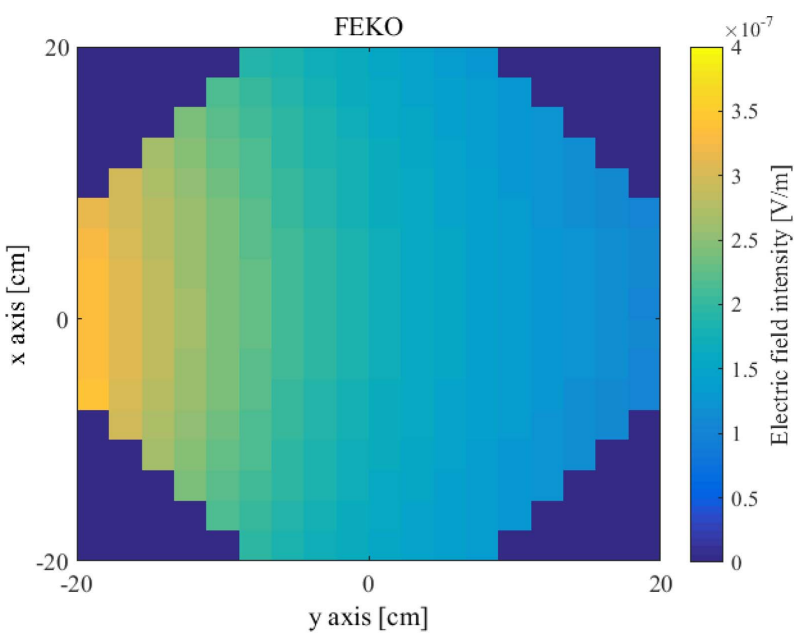

(a)

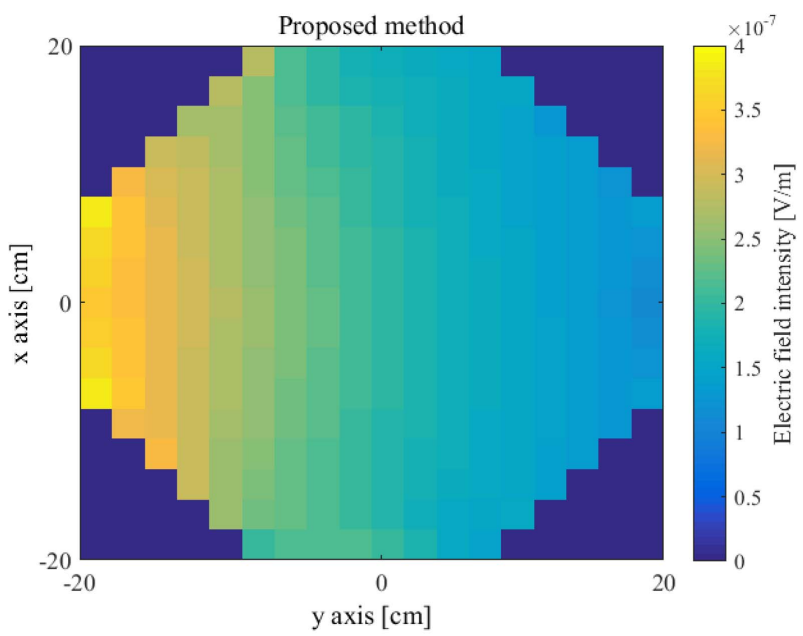

(b)

Fig. 6. (Color online) Absolute internal electric field crosssections from the helical antenna in the plane $\mathrm{z}=0$ : (a) FEKO, (b) the proposed method. 
Table 3. Induced electric field comparisons for the WPT system.

\begin{tabular}{lccc}
\hline \hline & \multicolumn{3}{c}{ Electric field } \\
\cline { 2 - 4 } & Plane $\mathrm{x}=0$ & Plane $\mathrm{y}=0$ & Plane $\mathrm{z}=0$ \\
\hline Maximum difference (\%) & 30.33 & 28.57 & 29.56 \\
Average difference (\%) & 10.52 & 9.27 & 10.30 \\
\hline
\end{tabular}

of the FEKO. The electromagnetic field computed by the two methods is compared with maximum and average relative difference and summarized in Table 2.

The internal electric field results obtained by the proposed method and by FEKO are shown in Fig. 6.

Finally, the proposed method is used to analyze the electric field induced from the WPT system. Two helical antennas with same parameter in the previous test are used. The distance between two antennas $\mathrm{D}_{\mathrm{W}}$ is $50 \mathrm{~mm}$.

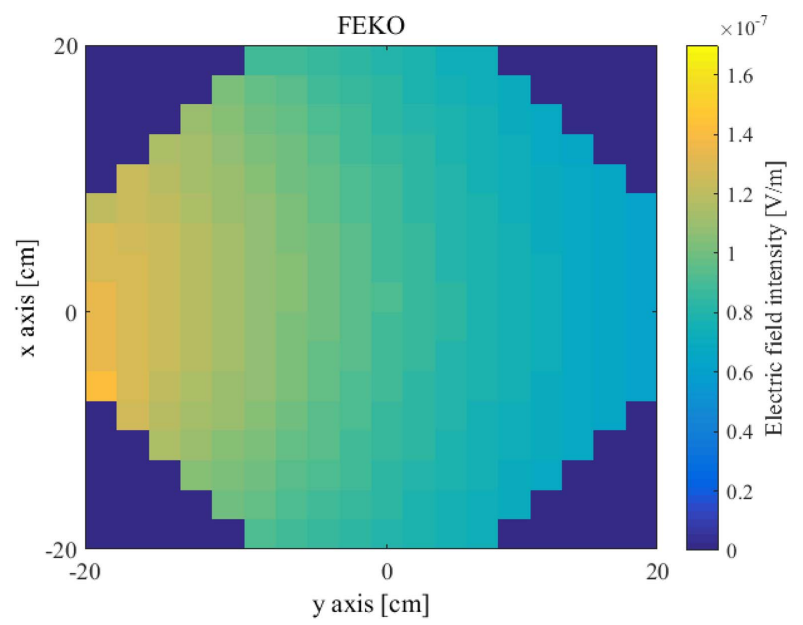

(a)

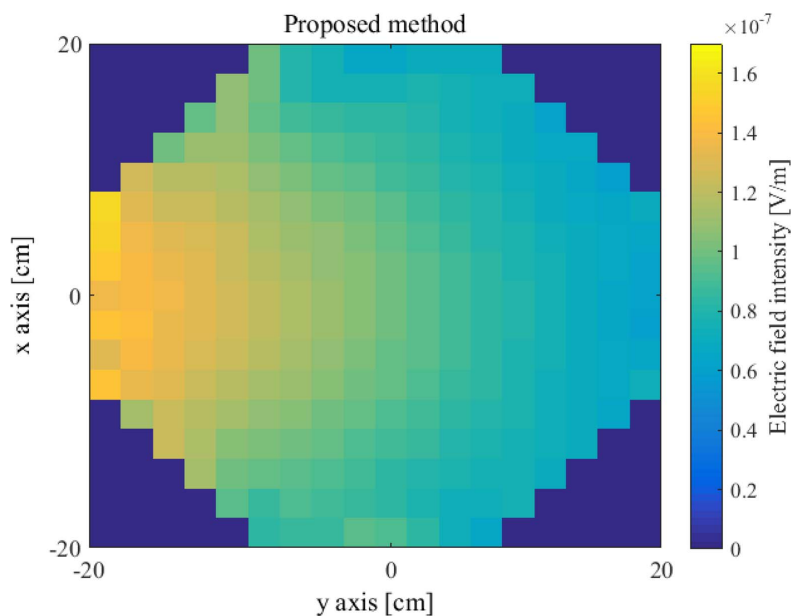

(b)

Fig. 7. (Color online) Absolute internal electric field crosssections from the WPT system in the plane $z=0$ : (a) FEKO, (b) the proposed method.
The other simulation setups were same as the previous test. The simulation rapidly reached steady-state in the same way as before two tests. The comparison result of the induced electric field is summarized in Table 3.

The internal electric field results obtained by the proposed method and by FEKO are shown in Fig. 7.

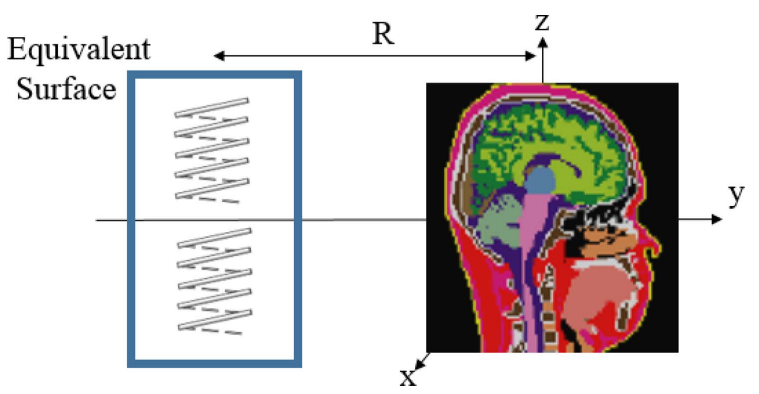

Fig. 8. (Color online) System for calculating the induced electric field of a realistic human head model from WPT system.

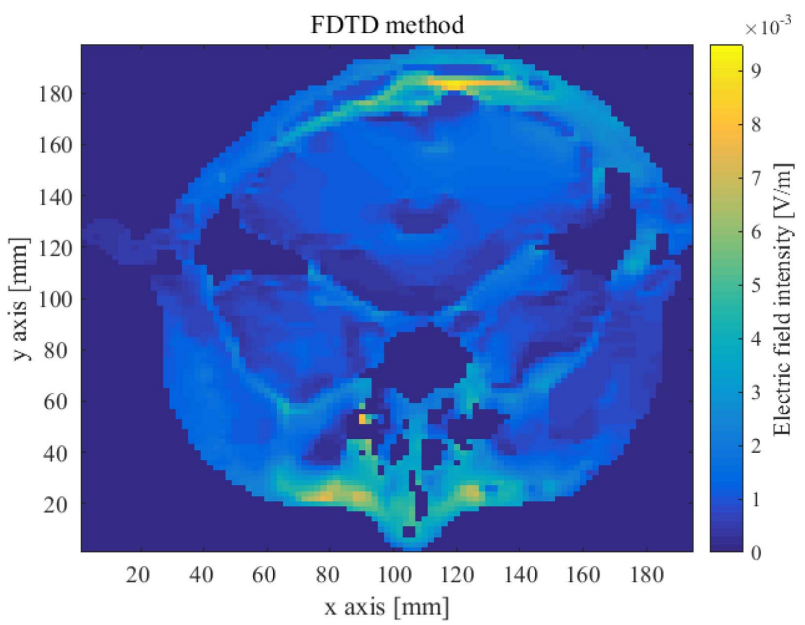

(a)

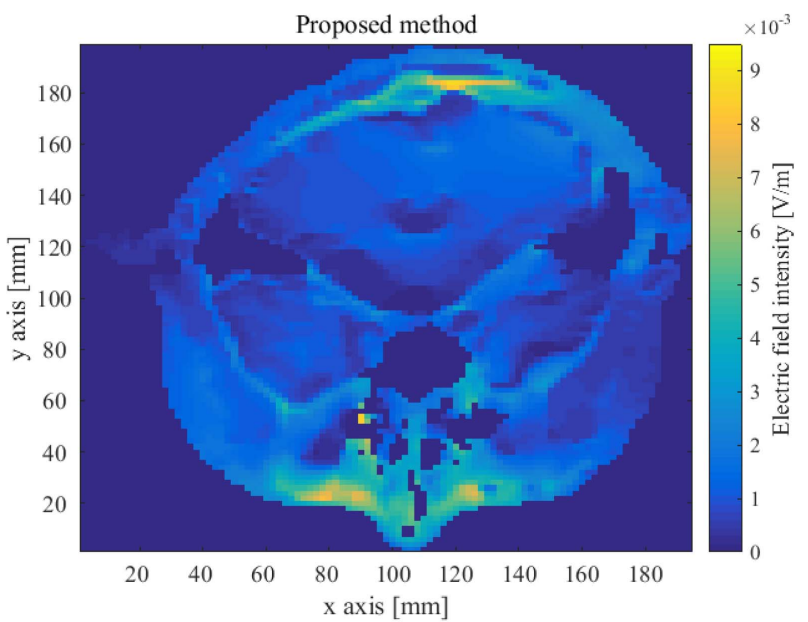

(b)

Fig. 9. (Color online) Absolute internal electric field crosssections from the WPT system in the plane $z=0$ : (a) FDTD method, (b) the proposed method. 
From the above results, the proposed method can be used to calculate the dosimetry of the WPT system, taking into account both the electric and magnetic coupling, in contrast to the conventional methods mentioned in the introduction. To demonstrate the computation improvement of the proposed method, a realistic human voxel model is analyzed. The standard FDTD method is selected for a comparison of the numerical technique, as it is widely used to solve bio-electromagnetic problems. The Japanese adult male model TARO [21] is analyzed for the dosimetry of the above WPT system. This model is composed of 51 tissues and organs with a spatial resolution of $2 \mathrm{~mm}$, based on accumulated magnetic resonance (MR) images. The electrical properties of this model are obtained from Gabriel's Cole-Cole models [22]. The WPT system is used as previous test. The center of the head part and the equivalent surface, including the WPT system, are located on the y-axis, as illustrated in Fig. 8.

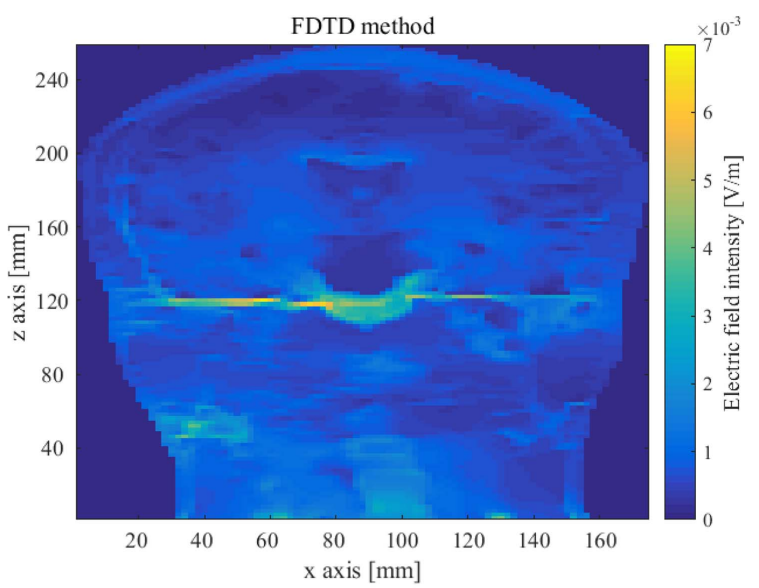

(a)

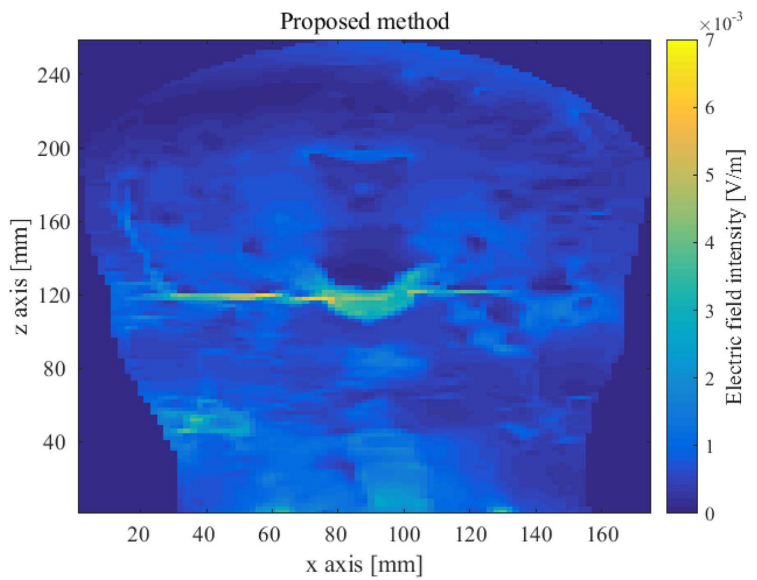

(b)

Fig. 10. (Color online) Absolute internal electric field crosssections from the WPT system in the plane $y=0$ : (a) FDTD method, (b) the proposed method.
The distance $\mathrm{R}$ between the center of the equivalent surface and the head part is $600 \mathrm{~mm}$. The two methods use an identical amount of memory, as they are based on a basic FDTD algorithm. The internal electric field results obtained by the proposed method and standard FDTD method are shown in Figs. 9, 10, 11.

The comparison results for the induced electric field are summarized in Table 4.

The maximum difference occurs associated with zero field values between two methods. These values are not crucial, however, as it is too small to affect for human exposure assessment.

The number of required iterations in the standard FDTD method for one period is determined as follows:

$$
N=\frac{\sqrt{n} \cdot c_{0}}{\text { frequency } \cdot \Delta x}
$$

Here, $n$ is the dimension of the simulation, $c_{0}$ is the speed of light, and $\Delta x$ is the mesh size [15].

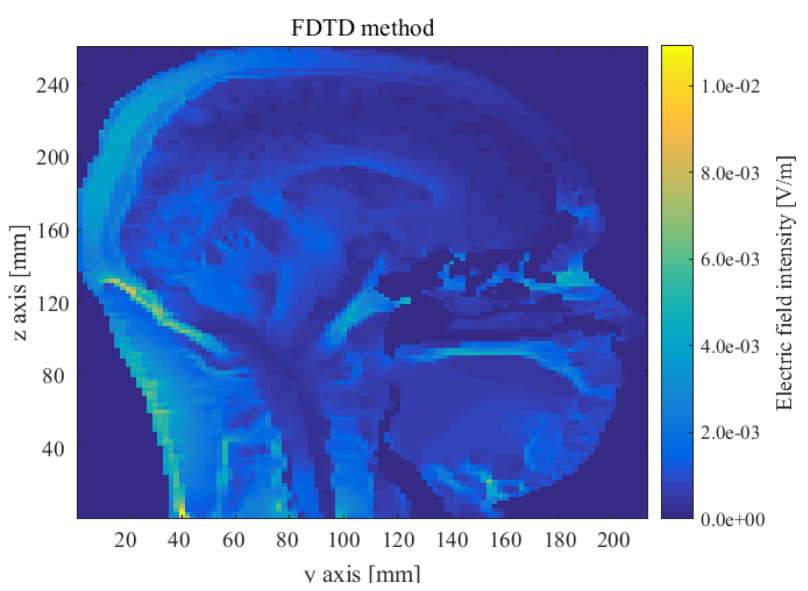

(a)

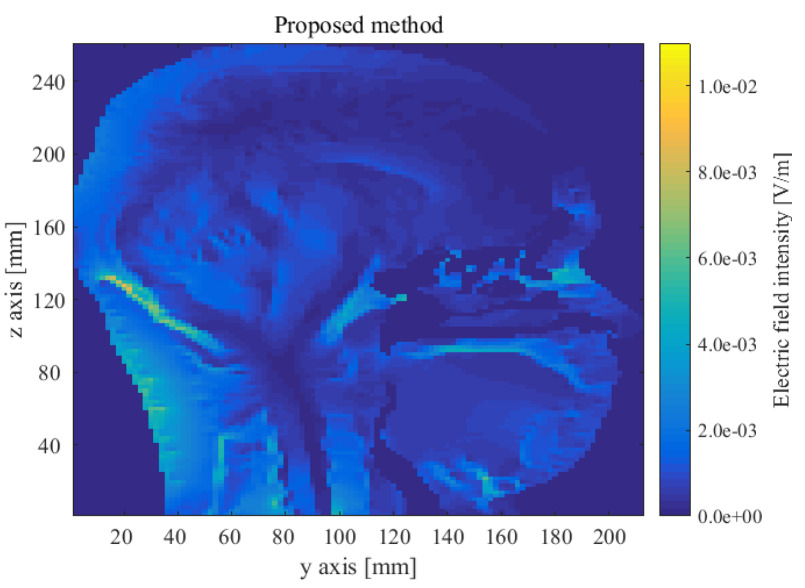

(b)

Fig. 11. (Color online) Absolute internal electric field crosssections from the WPT system in the plane $\mathrm{x}=0$ : (a) FDTD method, (b) the proposed method. 
Table 4. Induced electric field comparisons for the WPT system.

\begin{tabular}{lccc}
\hline \hline & \multicolumn{3}{c}{ Electric field } \\
\cline { 2 - 4 } & Plane $\mathrm{x}=0$ & Plane $\mathrm{y}=0$ & Plane $\mathrm{z}=0$ \\
\hline Maximum difference (\%) & 360.84 & 87.81 & 279.83 \\
Average difference (\%) & 11.05 & 9.98 & 5.65 \\
\hline
\end{tabular}

As this method typically requires a few periods of the source, the standard FDTD simulates the problem in three periods. This required nearly 865,440 time steps. The proposed method, however, converged after only 4000 time steps. Thus, the proposed method reduces the simulation time by nearby 215 -fold compared to the standard FDTD. It takes about 22.5 days and 2.5 hours with an Intel(R) Xeon ${ }^{\circledR}$ CPU E5-2650 v2 processor running at $2.60 \mathrm{GHz}$, respectively, for the two methods to finish the simulation. Moreover, the required iteration number $\mathrm{N}$ is increased when the frequency is lowered. Thus, the proposed method shows much stronger performance with low-frequency problems, as it reaches convergence rapidly regardless of any changes in the frequency.

\section{Conclusion}

We propose numerical method to analyze low-frequency problems in a short time regardless of the shape of the source using QSA, unlike the conventional methods. It is especially well suited for a heterogeneous dielectric and conductive body with arbitrary shaped scatterers. The internal electric fields of the dielectric sphere are calculated from the excitation of antennas to validate the SQFDTD method. Furthermore, the internal fields of the simplified human phantom model exposed to EMF from the WPT are also obtained and compared to those calculated by the commercial solver. Lastly, a simulation of a realistic human model is conducted as an example of the application of the proposed method. The results are in good agreement with those from the standard FDTD method. The proposed method is nearly 200 times faster than the convention method in a simulation at $1 \mathrm{MHz}$. Moreover, the proposed method is not affected by changing in frequency unlike the standard FDTD method. Our results successfully demonstrate the possibility of calculating the dosimetry from the WPT system with the proposed approach. We believe that the proposed method can be applied to the dosimetry of various electric machines operating at low-frequency band.

\section{Acknowledgement}

This research was supported by the Basic Science
Research Program through the National Research Foundation of Korea (NRF) funded by the Ministry of Education (NRF-2012R1A1A2006794).

\section{References}

[1] A. Kurs, A. Karalis, R. Moffatt, J. D. Joannopoulos, P. Fisher, and M. Soljačić, Science 317, 83 (2007).

[2] P. Stavroulakis, Biological effects of electromagnetic fields, Springer-verlag, Berlin (2003) pp. 187-190.

[3] T. Namiki, IEEE Trans. Microwave Theory Tech. 47, 2003 (1999).

[4] E. Li, I. Ahmed, and R. Vahldieck, IEEE Microw. Wireless Comp. Lett. 17, 319 (2007).

[5] S. Staker, C. Holloway, A. Bhobe, and M. Piket-May, IEEE Trans. Electromagn. Compat. 45, 156 (2003).

[6] J. Deford, H. Kanai, and O. P. Gandhi, IEEE Trans. Biomed Eng. 31, 644 (1984).

[7] J. Deford and O. P. Gandhi, IEEE Trans. Electromagn. Compat. EMC-27, 168 (1985).

[8] K. R. Davey, C. H. Cheng, and C. M. Epstein, IEEE Trans. Biomed. Eng. 38, 418 (1981).

[9] T. W. Dawson, J. D. Moerloose, and M. A. stuchly, Appl. Comput. Electromagn. Soc. J. 11, 63 (1996).

[10] S. W. Park, K. Wake, and S. Watanabe, IEEE Trans. Microw. Theory Techn. 61, 2153 (2013).

[11] O. P. Gandhi and J. Y. Chen, Bioelectro. 1, 43 (1992).

[12] J. D. Moerloose, T. W. Dawson, and M. A. Stuchly, Radio Sci. 32, 329 (1997).

[13] Electromagnetic Software and Systems (EMSS). FEKO, Stellenbosch, South Africa, 2011. [Online]. Available: http://www.feko.info/

[14] A. Elsherbeni and V. Demir, The finite-difference timedomain method for electromagnetics with matlab simulations, Scitech, Danver (2009) pp. 272-273.

[15] D. M. Sullivan, Electromagnetic Simulation Using The FDTD Method, IEEE Press. New York (2000) pp. 1-4.

[16] J. D. Jackson, Classical Electrodynamics, Wiley, Newyork (1999) pp. 218-220.

[17] W. T. Kaune, J. L. Guttman, and R. Kavet, Bioelectro. 18, 67 (1997).

[18] D. Andreuccetti and N. Zoppetti, Int. J. Occup. Saf. Ergon. 12, 201 (2006).

[19] A. Hirata, Y. Takano, and T. Nagai, IEICE Trans. E93-C 1, 60 (2010).

[20] M. E. Potter, M. Okoniewski, and M. A. Stuchly, J. Comput. Physics 162, 82 (2000).

[21] T. Nagaoka, S. Watanabe, K. Sakurai, E. Kunieda, S. Watanabe, M. Taki, and Y. Yamanaka, Phys. Med. Biol. 61, 1 (2004).

[22] C. Gabriel and S. Gabriel, Brooks AFB, San Antonio (2006). [Online]. Available: http://www.brooks.af.mil/AFRL/ HED/hedr/reports/dielectric/home.html 